\title{
Do your eye movements reveal your performance on an IQ test? A study linking eye movements and socio-demographic information to fluid intelligence.
}

Enkelejda Kasneci ${ }^{1 *}$, Gjergji Kasneci ${ }^{2}$, Ulrich Trautwein ${ }^{3}$, Tobias Appel ${ }^{3}$, Maike Tibus ${ }^{3}$, Susanne M. Jaeggi ${ }^{4}$, Peter Gerjets ${ }^{5}$

1 Human-Computer Interaction, Department of Computer Science, University of Tübingen, Tübingen, Germany

2 Data Science and Analytics, Department of Computer Science, University of Tübingen, Tübingen, Germany

3 Hector Research Institute of Education Sciences and Psychology, University of Tübingen, Tübingen, Germany

4 School of Education, University of California, Irvine, CA, USA

5 Leibniz-Institut für Wissensmedien, Tübingen, Germany

* Corresponding author: Enkelejda.Kasneci@uni-tuebingen.de

\section{Abstract}

Understanding the main factors contributing to individual differences in fluid intelligence is one of the main challenges of psychology. A vast body of research has evolved from the theoretical framework put forward by Cattell, who developed the Culture-Fair IQ Test (CFT 20-R) to assess fluid intelligence. In this work, we extend and complement the current state of research by analysing the differential and combined relationship between eye-movement patterns and socio-demographic information and the ability of a participant to correctly solve a CFT item. Our work shows that a participant's eye movements while solving a CFT item contain discriminative information and can be used to predict whether the participant will succeed in solving the test item. Moreover, the information related to eye movements complements the information provided by socio-demographic data when it comes to success prediction. In combination, both types of information yield a significantly higher predictive performance than each information type individually. To better understand the contributions of features related to eye movements and socio-demographic information to predict a participant's success in solving a CFT item, we employ state-of-the-art explainability techniques and show that, along with socio-demographic variables, eye-movement data. Especially the number of saccades and the mean pupil diameter, significantly increase the discriminating power. The eye-movement features are likely indicative of processing efficiency and invested mental effort. Beyond the specific contribution to research on how eye movements can serve as a means to uncover mechanisms underlying cognitive processes, the findings presented in this work pave the way for further in-depth investigations of factors predicting individual differences in fluid intelligence.

\section{Introduction}

With his theory of human intelligence published in 1963, Cattell [1] established a common understanding of two factors underlying human intelligence: crystallized and 
fluid intelligence. While crystallized intelligence primarily involves abilities related to acquired knowledge and experience, fluid intelligence encapsulates the general abilities of reasoning and problem solving regardless of such knowledge. As such, fluid intelligence is considered foundational to many cognitive tasks and, most importantly, to learning [18,46]. Therefore, researchers from various fields have investigated individual differences that contribute to fluid intelligence and - consequently - its derived skills in the areas of learning and cognition. Several approaches have been used to capture individual differences in fluid intelligence, including participants' reports of strategies [69] or motivational factors (e.g. effort 80,81$]$ ). Given that self-reports can be biased 82, psychophysiological measures, especially ocular movements like scanpaths 41] and pupil diameter [4] have shown to be useful indices for strategies and motivational factors to predict individual differences in fluid intelligence [51]. Thus, to paint a more complete picture of the factors contributing to an individual's performance in an intelligence test, eye tracking can play an important role as it enables researchers to investigate the participants' behavior in an unobtrusive way.

\section{Using eye movements to capture individual differences in fluid intelligence}

With the increasing availability of accurate and low-cost eye-tracking technology, new means of monitoring a participant during task accomplishment at a fine-grained level have become available. In particular, there has been an increased interest in employing eye movement analysis to improve understanding of the relationship between eye movements and the allocation of visual and cognitive resources. This interest is reflected in a considerable number of publications, especially in the fields of processing speed and working memory capacity, which are considered key processes underlying performance in fluid intelligence tests 48,49]. In this context, multiple research articles have also addressed the relationship between eye movements and performance in fluid intelligence measures through empirical studies [28, 40, 41, 44, 50, 51].

Prior research has also revealed the pupil to be a particularly interesting eye-related feature, since pupil size changes have been linked to task demands and cognitive effort 64. In their review, van der Wel and van Steenbergen argue that pupil diameter is an indicator for the general exertion of cognitive effort which tends to be higher for more difficult tasks 64]. Therefore, a more difficult task evokes a greater pupillary response indicating more mental effort and a greater mobilisation of cognitive resources. Already by 1979, Ahern and Beatty demonstrated that the link between task difficulty and pupil diameter in an arithmetic task is moderated by individual differences in intelligence [66]. Individuals with higher intelligence scores responded more accurately and demonstrated a smaller pupil diameter suggesting that individuals with higher IQ scores may need to exert less mental effort to successfully complete the task. In contrast, van der Meer reported an increase in pupil diameter and accuracy in participants with higher fluid intelligence scores solving an analogical reasoning task [43], but only for the most difficult item. Furthermore, Bornemann and colleagues have investigated 11th graders and found a significant positive correlation between task difficulty and pupil dilation for an analogy task that participants were unfamiliar with, but not for an algebra task that was already part of the curriculum [45]. They concluded that the novelty of the task allows participants with greater cognitive abilities to allocate more cognitive resources, whereas a familiar task does not cause this effect. Overall, there seems to be a complex interplay between task difficulty and pupil diameter that varies with task type, novelty, and intelligence.

A limited number of studies have investigated eye-movement patterns and strategies associated with performance in fluid intelligence tests. For example, in a study 
conducted by Vigneau et al., 51, 55 participants (i.e., university students) were monitored during 14 selected items of the Raven's Advanced Progressive Matrices test. The authors reported differences in viewing patterns between participants scoring relatively higher or lower on the test. Proportional time on the problem matrix (i.e., test item), latency to first alternation, and time distribution on the problem matrix were positively correlated to test scores, whereas the number of alternations between matrix and response choice and the gaze time spent on answer alternatives were negatively correlated to the test scores [51]. In particular, the authors argue that participants do not only differ with regards to their strategy, but also regarding how that strategy is employed, thereby adding a qualitative dimension to the existing distinction between constructive matching and response elimination 83 84. Similar findings were reported by Hayes et al., 40] in a study with 35 university students. The authors found that a significant percentage of the variance on the participants' scores on a Raven's Advanced Progressive Matrices test was explained by eye-fixation patterns, where systematic scanning of the problem matrices and less toggling between matrix and responses were indicative of better performance, which likely reflects differential strategies between groups: e.g., more successful participants might have constructed an internal representation of the problem before scanning the answer alternatives. Relatedly, Laurence et al. [3] investigated the association between eye movement patterns and IQ test performance in a study with 34 participants who completed a digitalized version of the Wiener Matrizen-Test 2. The authors reported that participants who scored higher on the test showed less gaze transitions between the relevant areas of interest and the response alternatives [3]. More recently, Sargezeh et al., 28] recorded eye movements of 44 participants while performing a comparative visual search task and found significant differences between participants who scored low, medium, and high on a measure of fluid intelligence using multiple features extracted from eye movements. More specifically, the authors reported a strong positive correlation between saccade peak velocity and test scores, while the ratio of total fixation duration to total saccade duration was negatively correlated with performance 28. Finally, Curie et al. 78 used a larger sample of 137 participants to investigate the validity of a new visual analogical reasoning paradigm for populations with intellectual disabilities and found that both, problem-solving strategies as well as eye-tracking data explained individual differences in performance. Overall, eye movement patterns seem to be indicative of participants' strategies, which are associated with successful performance in fluid intelligence tasks.

Although several eye movement features have shown to be useful in predicting individual differences in performance during fluid intelligence tasks, previous research in this domain has often been restricted to relatively small sample sizes. Thus, studies with larger sample sizes are required to reveal robust and reliable results. Our study addresses this research gap and provides a comprehensive analysis of eye-movement features related to task performance using a standardized test of fluid intelligence, the CFT at the item level. Furthermore, there is limited research that has focused on participant strategy and their roles in explaining individual differences in problem solving success. Thus, a more comprehensive approach that relies on more fine-grained behavioral and physiological measures and that also includes socio-demographic factors as an additional source of variance is needed to achieve a deeper understanding of the underlying mechanisms contributing to cognitive performance.

\section{Socio-demographic factors and fluid intelligence}

Theoretical accounts from various disciplines including sociology, economics, and psychology as well as empirical evidence suggest a robust link between socio-demographic factors and intelligence. Specifically, socio-economic status that includes the education level of participants and their parents has shown to be 
significantly correlated with fluid intelligence 65, 67, which has been attributed to differential experiences and opportunities 70 71. For example, Kaufman et. al demonstrated the correlation between years of formal education and fluid intelligence in a stratified sample of 1125 adults ranging from 22 to 90 years of age [65]. They found the same correlation for crystallized intelligence and several academic skills. Finally, using a school cut-off design, Zhang et al. 72] found that first-graders outperformed their age-matched kindergarten peers in matrix reasoning, indicating that experiences related to schooling impacted the development of intelligence. Overall, accumulating research has highlighted the importance of educational experiences for cognitive development, which translates to individual differences in intelligence. In addition to educational experiences, other activities have shown to contribute to cognitive performance including various leisure activities such as physical exercise [73], playing computer games 74 75], and musical training [76. Collectively, a host of experiential factors have shown to contribute to individual differences in intelligence.

\section{Research goals}

With this work, we aim to advance the literature on individual differences in fluid intelligence by including both, eye-tracking data as well as socio-demographic variables to predict task performance at the item level using machine learning techniques. To address our aims, we rely on the TüEye $Q$ data set 68,79 collected from 315 university students performing a fluid intelligence test. While a sample size of 315 participants is unusually large in a typical eye movement study, it is rather small-scale compared to other data sets that focus on socio-demographic factors and those in the machine learning literature. While we have hypotheses that are rooted in the body of research on either socio-demography or eye tracking, investigating them jointly has an exploratory character when problem solving success is concerned.

To the best of our knowledge, this is the first study that provides a methodological foundation for the investigation of factors that contribute to individual differences in problem solving skills by relying on socio-demographic, eye movement, and physiological data. More specifically, our predictive model is based on the Gradient Boosting Decision Trees (GBDT) 34 algorithm, which allows us to go beyond conventional linear statistical methods that are restricted to simple relationships between the features and the target variable. The GBDT algorithm is an ensemble approach that makes use of simple decision trees as base learners. Since each tree added to the ensemble is different from the previous trees and focuses on the remaining error, the GBDT algorithm helps to reduce bias [36 37, which is very important for data sets of moderate size where the instance-related bias and the variability across instances can negatively influence a predictive model. A further advantage of the GBDT algorithm is that it is not vulnerable in the presence of collinearity and is, therefore, very well suited for processing behavioural and eye-tracking data in a holistic way.

\section{Materials and methods}

The TüEyeQ data set was recently published on Nature Scientific Data to enable researchers to freely access the experimental data 79]. Therefore, for a thorough description of the experimental setup and further details on the data, we refer the reader to the data set description as published in 79. In the following, we will briefly describe the data collection and processing steps of TüEyeQ relevant to this work.

More specifically, let $P$ be the set of participants and $I$ the set of items of a CFT

20-R fluid intelligence test. For a feature-based description $\mathbf{x}_{P}(p)$ of a participant $p \in P$ and a feature-based description $\mathbf{x}_{I}(i)$ of a CFT 20-R item $i \in I$, we aim to predict 
whether $p$ will correctly solve $i$, that is, we search for a mapping

$f: \operatorname{concat}\left(\mathbf{x}_{P}(p), \mathbf{x}_{I}(i)\right) \mapsto\{0,1\}$ that can correctly predict whether a participant $p$ will succeed or fail on an item $i$, where $\operatorname{concat}\left(\mathbf{x}_{P}(p), \mathbf{x}_{I}(i)\right)$ denotes the concatenation of the feature vector describing the participant and the feature vector describing the item. Note that $\mathbf{x}_{P}(p)$ can vary depending on whether we use only socio-demographic information to describe the participant, or only eye-movement data, or both.

For a detailed description of the features contained in the TüEyeQ data set, we refer to Table 2 and 79 .

\section{The TüEyeQ data set}

Study participants TüEyeQ contains data collected from 315 healthy participants (217, female, 94 male, 4 not stated; with an age mean of 23.272 years, SD 3.022) completing the CFT-R, and providing their socio-demographic and educational background characteristics, including information on leisure time activities and the use of technology, software, and gaming (see Table 2 for a complete list of variables). Due to technical shortcomings or low tracking quality, eye movement data is available for only 229 out of these 315 participants as will be explicitly described in the following. All participants had a university entrance qualification, reported no neurological or psychiatric pre-existing conditions, and no visual impairment above 3 dioptres. The Ethics Committee at the Psychological Institute at the University of Tübingen confirmed that the procedures were in line with ethical standards of research with human subjects. All participants were informed in written form and consented that their anonymous data can be analyzed and published. Due to a self-constructed pseudonym, they had the option to revoke this consent at any time.

The IQ test The participants performed the first part of the revised version of the "culture fair" intelligence test (CFT 20-R) designed by Weiß et al. [2]. This test is intended to measure the general mental capacity (i.e., the g-factor of intelligence or fluid intelligence) by means of different problem types that require the ability to recognize figural relationships and to engage in formal logical thinking in problems of varying degrees of complexity under a time restriction. The CFT 20-R consists of four categories of different problem types, namely series continuation, classifications, matrices, and topological conclusions. Each of these categories consists of 11-15 test items with increasing difficulty and a time limit of 3-4 minutes.

In order to record the eye movements of the participants during the task, we adapted the classic pen-and-paper version of the IQ test to a digital one that can be displayed on a computer screen. To imitate paper version as closely as possible, we presented several test items on a single screen page as long as this did not necessitate scrolling. Further information regarding the presentation and layout of the test can be found in [79].

Data acquisition As described in 79], the data was collected in a digital classroom equipped with 30 remote eye trackers attached to laptops with 17inch HD display screens running at full brightness. This setup allows for data collection of up to 30 participants simultaneously, minimizing the overall time needed for collection. For this study, verbal instructions were given to the entire group pertaining to a brief overview of the protocol and an explanation of eye tracking, then individual calibrations were performed with a supervised quality check. Interactions between the participants and the computer took place via mouse or touch pad depending on participants' preference.

The collection environment controlled the room illumination level, ensuring no effects from sunlight or other outdoor light. The standard maintained illuminance for 
the experimental sessions was between 10 to 50 lux, measured with a Lux sensor (i.e., Gossen Mavo-Max illuminance sensor, MC Technologies, Hannover, Germany).

The study participants first received general instructions about the nature of the test, followed by the first block consisting of one particular type of problem. Each block had specific instructions, introducing the participants to the block's requirements and demonstrating the essence of the problem types based on three exemplary test items. The instruction phase was conducted without time constraints, thus all participants could go through the examples and familiarize themselves with the test procedure. All instructions were presented in German using the SoSci Survey online platform [4].

Eye-tracking equipment Eye movement data were collected by means of SMI RED250 remote eye trackers, a commercial eye tracker with $250 \mathrm{~Hz}$ sampling frequency. Since the eye tracker has a high sampling frequency, both stable (fixations) and rapid (saccadic) eye movements for static stimuli can be measured. Eye movements were recorded using the included eye-tracking software Experiment Center which outputs the raw gaze data consisting of $\mathrm{x}$ and $\mathrm{y}$ coordinates of each data point, the timestamp information, and the pupil diameter in millimeters.

Calibration was performed for all participants. A validation also was performed as a quality check to measure the gaze deviation for both eyes from a calibration point. A deviation larger than one degree required re-calibration. Calibrations were performed prior to the experiments as well as one or two times during the experimental session depending on how many images were presented.

Quality of eye tracking data Initially, the raw gaze data was examined for signal quality using the eye-tracking software BeGaze provided along with the eye trackers. This software reports the proportion of valid gaze signal to stimulus time as the tracking ratio. Therefore, if a participant's tracking ratio was deemed insufficient (i.e., less than $80 \%$ for at least a part of the task), we omitted the corresponding eye-tracking data. This pre-processing stage can assure that errors (e.g. post-calibration shifts, poor signal due to glasses) in the gaze data are substantially minimized. Consequently, eye-tracking data from 58 participants had to be omitted due to low tracking ratios. An additional 11 eye-tracking data sets were excluded due to errors in the presentation software and another 17 because of incomplete data. This leaves us with eye-tracking data for only 229 of 315 participants. The raw eye-tracking data was then pre-processed to improve the data quality and to extract the relevant features.

Eye movement features Building on previous work that has focused on the investigation of individual differences in fluid intelligence using eye-movement data, our aim was to uncover the extent to which specific eye-movement features can predict CFT performance at the item level. More specifically, the selection of eye-movement features used in our work is informed by previous work; that is, we focus on such features that have shown to be indicative of specific strategies during problem solving as discussed in the introduction. Beyond the typically used features, e.g., fixation or saccade related features, we also include some additional features (e.g. microsaccades, pupillometry) that are more exploratory. Following eye-movement features as provided by the TüEyeQ data set were considered in our models:

- Fixation-related information - Fixations describe the periods where the eye is "still" and thus perceiving visual information. Fixations were extracted from the raw eye-tracking data based on the I-VT algorithm 27. In our models, we use both the number of fixations, i.e., fixationCount, as well as their mean duration, i.e., meanFixationDuration, during an IQ-test item, since previous work relates longer fixations to higher processing load and more effort [15]. 
- Microsaccades-related information - Microsaccades, i.e., fixational eye movements, occur during an especially prolonged fixation, and have been previously linked to visual attention [5] 6], perception [7], working memory [8], and task difficulty [9]. In this work, we used the following features related to microsaccades from the TüEyeQ data set: microsaccadeCount (i.e., the mean number of microsaccades that occurred during a particular CFT task performance), meanMicrosaccadeAmplitude (i.e., the mean amplitude of microsaccades that occurred during each item of the CFT), meanMicrosaccadeDuration (i.e., the mean duration of microsaccades that occurred during problem solving), meanMicrosaccadePeakVelocity, (i.e., the mean peak velocity of microsaccades during problem solving).

- Saccade-related information - The TüEyeQ data set also provided saccade-related information. Saccades are rapid eye movements that enable us to change the focus of attention and were extracted from the eye-tracking protocols based on the I-VT algorithm 27] with a velocity threshold of $30^{\circ} / \mathrm{s}$. Since saccade velocity depends on neural activity and cannot be voluntarily controlled 29], saccade parameters have been previously linked to fluid intelligence. In our analysis, we employ the following parameters of saccades from TüEyeD: saccadeCount (corresponding to the number of saccades occurring during problem solving), the meanSaccadeAmplitude ((i.e., the mean amplitude of saccades occurring during problem solving), and the meanSaccadeDuration (i.e., the mean duration of saccades accruing during problem solving).

- Pupillary information - Since pupil diameter has been used used as an indicator of cognitive load, short-term memory, language processing, reasoning, perception, sustained attention, and selective attention [10,21,22, 30 33, 77, we also include the mean pupil diameter in our data analysis (meanPupilDiameter) and explicitly investigate its role in determining problem solving success.

\section{Feature and factor analysis}

Table 2 provides a detailed description of all features provided by the TüEyeQ data set. In addition to that, Figure 1 shows the percentage of correct responses for each test item and the average success rate (i.e., across all items in purple).

To analyse the socio-demographic and eye-movement features with respect to their variability, we first removed the columns related to the following features from the data set: participant (i.e., the id of a participant), taskID (i.e., the id of an item ${ }^{1}$ in the CFT 20-R), cft_task (i.e., a binary variable indicating whether the CFT item was solved correctly), and cft_sum_full (i.e., the total score of a participant over the CFT $20-\mathrm{R}$ ). After removing these features, we were left with a total of 85 features, 10 of which are eye-movement features.

In a next step, we developed a predictive model on the TüEyeQ data set with the goal of reliably predicting the performance of a participant on a random CFT item as described by its taskID, which gives hints at both the item type (i.e., one of the four task blocks of the CFT 20-R framework) and the position of the item within the corresponding CFT 20-R task block (i.e., of items of the same type). An increasing index of taskID (i.e., as represented by the ending digits) corresponds to increasing task difficulty.

In this work, we also conduct a feature importance analysis on the prediction of a participant's CFT task performance and find that some features are, indeed, highly

\footnotetext{
${ }^{1}$ Note that the feature taskID encodes both the CFT 20-R category/block to which the task belongs and the position of the task within that category.
} 


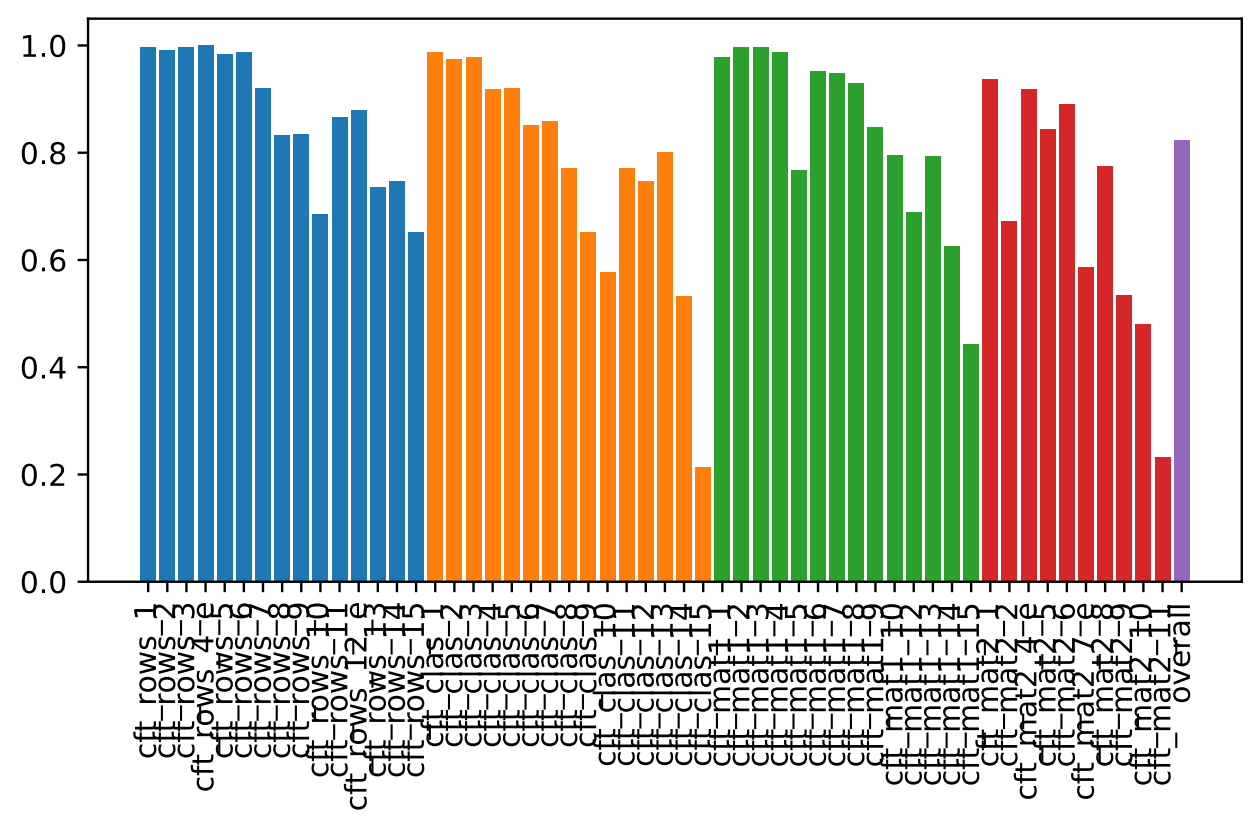

Fig 1. Success rates for each item of the CFT and a summary regarding all responses combined. The purple bar (on the very right) stands for the average success rate across all test items. The remaining colors encode the four different blocks of the test.

discriminative from a statistical point of view.

\section{Predicting task performance: Model description}

To identify an adequate predictive model for the TüEyeQ data, we conducted an empirical evaluation of various machine learning algorithms on the data. Not surprisingly, a predictive model based on the Gradient Boosting Decision Trees (GBDT) algorithm [34] showed the highest predictive performance. Our empirical findings on the excellent predictive performance of GBDT are also supported and complemented by previous results from numerous Data Science competitions and challenges. More specifically, according to [35, among the 29 winning solutions of Kaggle challenges ${ }^{2}$ in 2015, 17 solutions used the GBDT algorithm.

The GBDT algorithm is an ensemble approach that makes use of simple decision trees as base learners. A new decision tree $t_{k}$ is added at step $k$ to optimize $L^{k}=\sum_{j=1}^{n}\left(y_{j}-\left(t_{k}\left(\mathbf{x}_{j}\right)+\hat{y}_{j}^{k-1}\right)\right)^{2}+\sum_{i=1}^{k} \Omega\left(t_{i}\right)$, where $n$ is the number of training instances, $y_{j}$ is the true label of $\mathbf{x}_{j}, \hat{y}_{j}^{k-1}$ denotes the prediction for $\mathbf{x}_{j}$ based on the $k-1$ decision trees used so far, and $t_{k}$ represents the new decision tree. $\Omega(\cdot)$ is a regularisation term, which imposes constraints on the tree structures. The above loss can be minimized through stochastic gradient descent hence the name of the approach. Interestingly, the above loss function can be reformulated in a way that yields a clear strategy for the growing procedure of the current tree (i.e., whether or not to continue splitting a node, which feature to use, etc.).

Since each tree added to the ensemble is different from the previous trees and focuses on the remaining error, the GBDT algorithm helps to reduce bias [36.37, which is very important for data sets of moderate size where the instance-related bias and the variability across instances can negatively influence a predictive model. Another

${ }^{2}$ https://www.kaggle.com/competitions 
advantage of the GBDT algorithm is that it is not vulnerable in the presence of collinearity and is, therefore, very well suited for processing behavioural and eye-tracking data. Moreover, the GBDT algorithm has high application value since it can deal effectively with missing values and does not require much data preprocessing (apart from turning the target variable into a nominal variable). Note that this is a strong advantage over other advanced ML algorithms (e.g. Deep Learning) because most of the information contained in the data can be maintained, which is highly beneficial to the prediction quality.

For the development of our GBDT-based model, we used the LGMBClassifier from the LightGBM ${ }^{3}$ framework. Our model employed 100 decision trees with a maximum depth of 7 for each tree, a learning rate of 0.1 , and a scale_pos_weight parameter that compensates for the class imbalance in the dataset. All other parameters were left in their default configuration. The script that was used in our analysis is provided as a supplementary file to ensure transparency and reproducibility of our results.

Validation To provide robust estimations and exploit the training data as effectively as possible, we adopted a stratified group 20 -fold cross-validation strategy. Thus, the largest part of the data (i.e., over 16.000 instances) was used for training and other 800 examples for testing. It also ensured that data from any participant is only ever present in either the training or the validation set. Note that although there is some variance in the predictive performance across the 20 folds, as can be seen in the Figures $3 \mathrm{a}$, 3b and $3 \mathrm{c}$, the standard deviation of the ROC curves is within an acceptable range.

ROC-AUC measure The ROC-AUC (i.e., the area under the receiver operating characteristic curve) quantifies the performance of a classification model over all classification score thresholds. The ROC curve plots two parameters: (1) the True Positive Rate, i.e., $t p r=\frac{T P}{T P+F N}$, and (2) the False Positive Rate, i.e., $f p r=\frac{F P}{F P+T N}$. Note that the $t p r$ is a synonym for the recall of a predictive algorithm whereas the $f p r$ represents the rate of false alarms. The ROC curve plots the $t p r$ vs. the fpr values at different classification score thresholds. It can be shown that the area under the ROC curve is the ranking accuracy with respect to the classification score returned by a classifier. Ideally, instances that belong to the positive class should be assigned a higher score by the classifier and thus ranked higher than the instances that belong to the negative class. Hence, an AUC of 1 means that all positive instances are ranked before the negative instances and the two classes are clearly separated by the classifier. In contrast, an AUC of 0.5 means that there is no order across the instances and, as a result, the classes cannot be separated.

\section{Explainability approach}

In Machine Learning, post-hoc explanability techniques can help gain insight into the importance of input features for predictions made by a complex model $f: \mathbb{R}^{n} \rightarrow[0,1]$ (e.g., an ensemble model that uses $n$ different input features like the GBDT model described earlier). Two of the most popular techniques, so-called local attribution frameworks, are described in $24-26$. The main idea behind local attribution explainability is to generate a local attribution score for each feature by optimizing a simple (typically linear) explanation model $g$ such that it locally approximates the complex model $f$. Hence, $g$ can be seen as a local interpolation of $f$ in the region of interest, i.e., in the close neighborhood of an input $\mathbf{x} \in \mathbb{R}^{n}$, where $n$ is the number of input features.

\footnotetext{
${ }^{3}$ https://lightgbm.readthedocs.i0
} 
One of the most widely used local attribution techniques that comes with a strong semantic interpretation of a feature's importance was introduced in 24,25]. It

approximates the Shapley value $[52$ of a feature to quantify it's local attribution score. The Shapley value originates from Cooperative Game Theory and is a value that represents a player's contribution to the result achieved by a coalition of players. In terms of predictive modelling, the Shapley value determines the marginal contribution of an input feature to the prediction for all possible combinations of inputs. Specifically, according to the original formalisation in [52], given a feature vector $\mathbf{x}=\left(x_{j}\right)_{j=1}^{n}$, let $\phi_{j} \in \mathbb{R}$ be the Shapley value of input feature $x_{j} \in \mathbb{R}$ :

$$
\phi_{j}=\sum_{S \subseteq F \backslash\{j\}} \frac{|S| !(|F|-|S|-1) !}{|F| !}\left[f_{S \cup\{j\}}\left(x_{S \cup\{j\}}\right)-f_{S}\left(x_{S}\right)\right],
$$

where $F$ denotes the original set of features and $|\cdot|$ denotes the cardinality of a set. Besides, $f_{S}\left(x_{S}\right)$ is the prediction of model $f$ based on the input features that are included in the subset $S$. For practical purposes, the other features (i.e., in $F \backslash S$ ) are not removed; instead they are set to baseline values [53. We can reformulate (1) in terms of a characteristic function $v(S)$ to express $\phi_{j}$ as the expectation of the marginal contribution of feature $x_{j}$ :

$$
\phi_{j}=\sum_{S \subseteq F \backslash\{j\}} v(S \cup\{j\})-v(S)=\sum_{S \subseteq F \backslash\{j\}} \Delta_{j} v(S)=\mathbb{E}_{S}\left[\Delta_{j} v(S)\right]
$$

For the computation of the Shapley value, we would have to consider $2^{|F|}$ feature subsets, which is not feasible for high-dimensional data. Hence, various approximations of (1) have been proposed 24,57,58. For the explainability analysis in this work, we employ the TreeExplainer approach presented in 24 .

\section{Results}

In this section, we first report the results of the statistical tests on the eye-movement features. More specifically, we investigated the differences between participants either solving the item correctly ("task solved", representing one item within the CFT) or answering the item incorrectly ("task not solved") using all eye-movement features provided by the TüEyeQ data set. We further examined the predictive information as drawn from the socio-demographic features, eye-movement features, as well as from the combination of all features, respectively. Finally, we investigate the differential impact of the implemented features on the prediction made by the machine learning model.

\section{Eye-movement data}

In a first step, we conducted statistical tests with regard to the eye-movement information in the TüEyeQ data set. More specifically, a t-test to compare items that were solved correctly and those that were answered incorrectly. The results of this statistical comparison are shown in Table 1.

As presented in Table 1 and shown in Fig. 2a, our results show a highly significant difference $(p<0.0001)$ between fixation counts on items that were answered incorrectly $(29.07 \pm 21.71)$ and and those that were solved correctly $(17.16 \pm 13.89)$. More specifically, CFT items that were solved correctly were characterized by significantly less fixations than CFT items that were incorrectly answered by the participants. Our results show similar findings with regard to the mean fixation duration (Fig. 2b), indicating significantly shorter fixations for correctly solved CFT items 
Table 1. Statistical comparison of the eye-movement features during items that were solved correctly vs. those that were answered incorrectly

\begin{tabular}{|c|c|c|c|c|c|c|}
\hline \multirow[t]{2}{*}{ Eye-movement feature } & \multirow{2}{*}{$\begin{array}{l}\text { Cohen's d } \\
\text { correct } \\
\text { vs. } \\
\text { incorrect }\end{array}$} & \multirow{2}{*}{$\begin{array}{l}\text { p-value } \\
\text { correct } \\
\text { vs. } \\
\text { incorrect }\end{array}$} & \multicolumn{2}{|c|}{$\begin{array}{l}\text { Incorrectly } \\
\text { answered }\end{array}$} & \multicolumn{2}{|c|}{$\begin{array}{l}\text { Correctly } \\
\text { solved }\end{array}$} \\
\hline & & & Mean & SD & Mean & SD \\
\hline fixationCount & -0.65 & $\leq 10^{-161}$ & 29.07 & 21.71 & 17.16 & 13.89 \\
\hline meanFixationDuration $[\mathrm{ms}]$ & -0.13 & $\leq 10^{-5}$ & 582.07 & 243.81 & 549.95 & 262.63 \\
\hline saccadeCount & -0.66 & $\leq 10^{-163}$ & 29.76 & 22.20 & 17.20 & 13.99 \\
\hline meanSaccadeAmplitude $[\mathrm{px}]$ & 0.10 & $\leq 10^{-4}$ & 223.70 & 75.58 & 230.94 & 75.52 \\
\hline meanSaccadeDuration [ms] & 0.04 & 0.12 & 28.08 & 4.97 & 28.28 & 4.80 \\
\hline microsaccadeCount & -0.02 & 0.51 & 1.91 & 1.31 & 1.89 & 1.39 \\
\hline $\operatorname{mpanM}_{[\mathrm{px}]}$ & -0.02 & 0.53 & 7.47 & 7.14 & 7.34 & 7.66 \\
\hline$\prod_{[\mathrm{ms}]}^{\mathrm{meanMicrosaccadeDuration}}$ & -0.02 & 0.51 & 11.48 & 7.85 & 11.33 & 8.36 \\
\hline$\underset{[\mathrm{px} / \text { frame] }}{\operatorname{meanMicrosaccadePeakVelocity}}$ & -0.02 & 0.43 & 23.48 & 18.76 & 23.04 & 19.43 \\
\hline meanPupilDiameter $[\mathrm{mm}]$ & 0.14 & $\leq 10^{-6}$ & 3.38 & 0.33 & 3.43 & 0.33 \\
\hline
\end{tabular}

(549.95ms $\pm 262.63 \mathrm{~ms})$ than for items that were answered incorrectly (582.07ms \pm 243.81$)$.

Consistent with the previously described data, we found highly significant differences regarding the saccade-related features SaccadeCount (i.e., the number of saccades) and meanSaccadeAmplitude. As shown in Table 1 and Fig. 2c in the case of correctly solved CFT items, the participants performed significantly less saccades than for test items that were incorrectly answered. Furthermore, during CFT items that were solved correctly, participants performed saccades with significantly larger amplitudes than during items that were incorrectly answered, see also Fig. 2d. With regard to the feature MeanSaccadeDuration, we found no significant difference between the two conditions. As shown in Table 1 and in the Figures 2f, 2g, 2h, and 2i there were no significant differences for miccrosaccade-related parameters between the two behaviours (task-solved vs. task not solved).

With regard to the pupil diameter size, we found a highly significant difference between the the items that were solved correctly and those which were not. As shown in Table 1, participants showed a larger pupil diameter size in the case of correctly solved test items as compared to those items that were answered incorrectly.

\section{Predictive information in the features}

Our goal was to evaluate the impact of the features related to eye-movements on predicting whether a participant successfully solved a given CFT item. To this end, we built three GBDT models with the same number of decision trees, i.e., 100, the same maximum depth of 7 per tree, and the same learning rate of 0.1. All three GBDT models were trained and validated by applying the cross-validation procedure as introduced above in the model description.

The first GBDT model was trained on the eye-movement-related features only. The second GBDT model was trained only on the socio-demographic features, and the third was trained on the combined 85 features. There are two questions of interest:

- Are the features related to eye movements informative enough for predicting the performance of a participant on a given CFT item? 


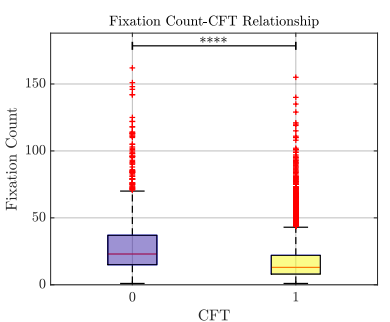

(a) Fixation Count

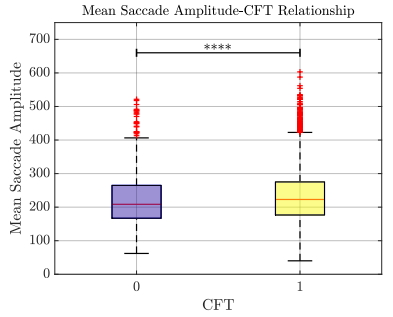

(d) Mean saccade amplitude

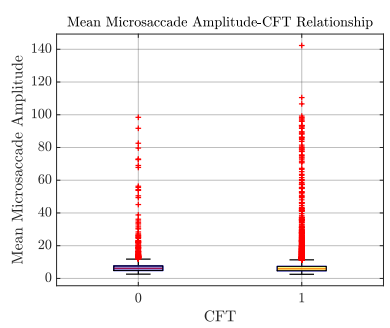

(g) Mean Microsaccade Amplitude

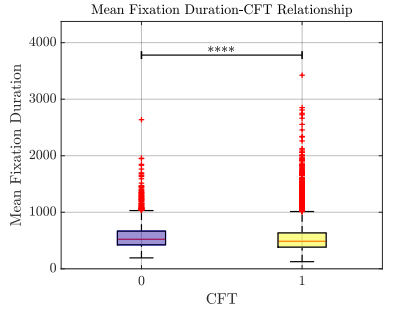

(b) Mean Fixation Duration

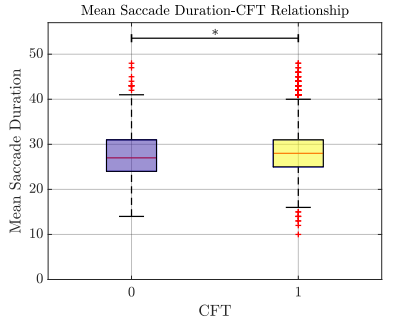

(e) Mean saccade duration

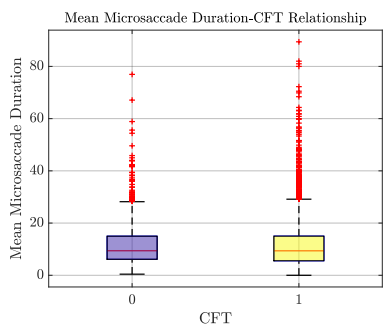

(h) Mean Microsaccade Duration

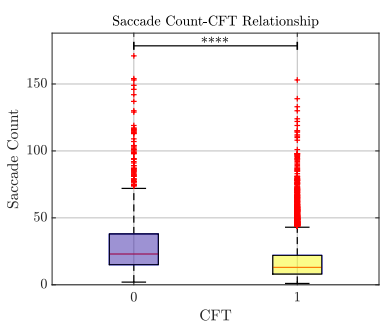

(c) Saccade count

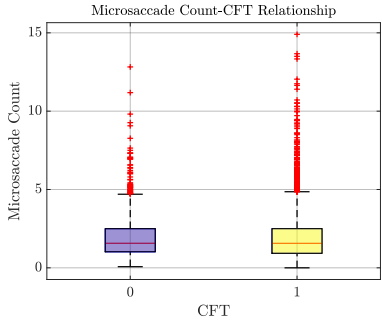

(f) Microsaccade Count

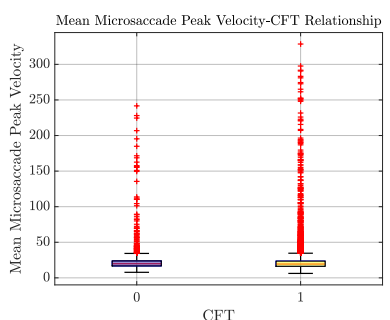

(i) Mean Peak Velocity of Microsaccades

Fig 2. Eye movement differences between the incorrectly answered (purple) and correctly solved items (yellow).

- If so, is the information contained in the eye-movement features complementary to the information contained in the socio-demographic features? Or, more specifically, is a predictive model developed on both types of features, i.e., the eye-movement and the socio-demographic features, more discriminative than the models developed on the single subgroups of features?

Interestingly, as it can be seen in Figures 3a, the GBDT model developed on the eye-movement features alone is already discriminative with an ROC-AUC of 0.63. Note that the model uses only 10 features (i.e., the features related to eye-movements in the TüEyeQ data set). The GBDT model developed on the 75 socio-demographic features is, as shown in Figure 3b less discriminative, with an ROC-AUC of 0.56. However, as depicted in Figure $3 \mathrm{c}$, the socio-demographic and the eye-movement-related features contain complementary information, and thus, the GBDT model developed on all features is the most discriminative, with an ROC-AUC of 0.65 . Note that this difference with regard to the discriminative performance of the model is substantial, and thus, highlighting the complementary contribution of eye movement features and socio-demographic features to the predictive model. 


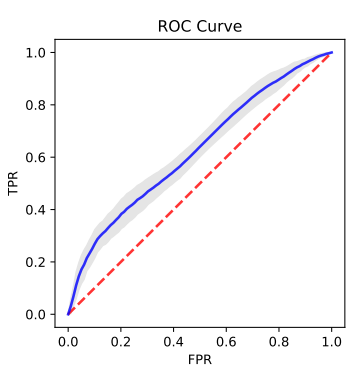

(a) AUC of 0.63

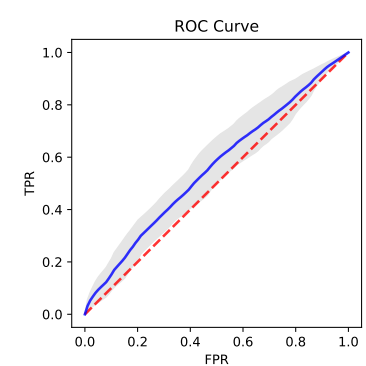

(b) AUC of 0.56

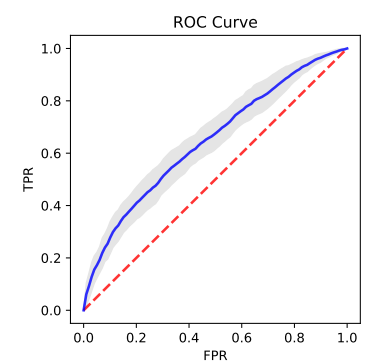

(c) AUC of 0.65

Fig 3. ROC curves of the GBDT model on the TüEyeQ data set that was trained on (a) only the features related to eye-movements, (b) only the socio-demographic features, and (c) on the socio-demographic and eye-movement-related features. Standard deviations based on the 20 folds of cross-validation are shown in gray.

\section{Explainability results}

Figure 4 shows that the features saccadeCount and meanPupilDiameter followed by meanFixationDuration and fixationCount provide the maximum information according to their marginal contribution density for the GBDT model that uses only the eye-movement features. It also becomes apparent that less saccades, a lower mean fixation duration and a larger mean pupil diameter contribute to the success of solving a item of the CFT.

Figure 5 shows that the features grades_math, mean_grade_degree, online_news_usage followed by background information on digital affinity and parental education/jobs provide the maximum information according to their marginal contribution density for the GBDT model that uses only socio-demographic features. Good grades in mathematics and participants' current standing in their study subject seem to indicate better performance in the CFT, while most forms of media consumption imply the contrary.

In Figure 6 we can see that five of the seven most informative features are eye-movement related - with grades_math and mean_grade_degree being the exceptions. The feature importances regarding eye movements are very similar to the ones already shown in Figure 4 and again indicate that saccade count, pupil diameter, and fixation duration carry information about the success while solving individual test items. Additionally, participants' primary subject of study and their parents occupation play an important role as highlighted by their distributions. This is also in line with the importances that were presented in Figure 5 .

\section{Discussion}

Following the structure of the result sections, we will first discuss our findings from the statistical tests on the eye-movement features, followed by a discussion on the predictive power of eye movements, socio-demographic information, as well as the overall combined feature set, respectively.

\section{Statistics of the eye-movement features}

Fixation-related features As presented in Table 1 and Fig. 2a, both fixations-related features (i.e., fixation count and mean fixation duration) showed that there was a highly significant difference between those items that were solved correctly 


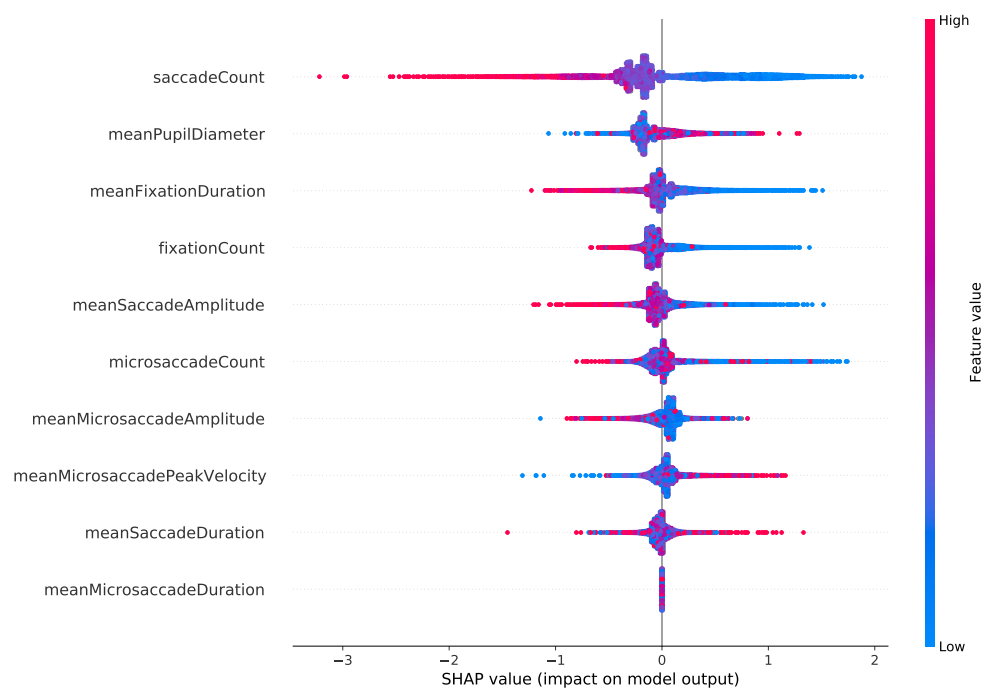

Fig 4. Summary plot of the approx. Shapeley values, that is, the density of the marginal contributions of the features in the GBDT model that uses only the eye-movement features. Red denotes high feature values, whereas blue indicates low feature values, and grey show categorical values or missing values that cannot be assigned a feature value.

and those which were not. During CFT items that were solved correctly, participants showed fewer fixations as compared with test items that were answered incorrectly, indicating faster processing speed and higher confidence. Additionally, correctly solved CFT items were characterized by shorter fixation durations, indicating more confidence in extracting and processing (visual) information. Our results are well in line with previous work that has reported associations between fixation duration and memory and processing load 55 56] 28. Furthermore, they support the general literature that has emphasized the relationship between fixation properties and performance in executive function and fluid intelligence tests, e.g., [12, 28, 54.

Saccade-related features As reported in Table 1, participants performed significantly less saccades for test items that were correctly solved than for those CFT items that they answered incorrectly. Additionally, in the case of correctly solved items, the participants performed saccades with significantly larger amplitude and longer duration. These results are in line with findings from related work [3, indicating an efficient visual search strategy, and/or successful retrieval of mental representations 19. Along this line, Sargezeh et al., 28 also reported a strong positive correlation between saccade peak velocity and performance in fluid intelligence tasks [28].

Microsaccade-related features With regard to the microsaccade-related features (i.e., the mean number of microsaccades, the mean microsaccade amplitude and duration, and the mean peak velocity of microsaccades) we found no significant differences between the CFT items solved correctly and those that were answered incorrectly. Although the relationship between microsaccades, working memory [8, 16], and task difficulty $[9]$ is gaining increasing research interest, our results did not show significant differences between the items solved vs. items not solved with regard to these features, despite the fact that the items that were answered incorrectly were generally more difficult. There might be different explanations for these results. First, the eye-tracking data provided by the TüEyeQ data set was captured at $250 \mathrm{~Hz}$, which 


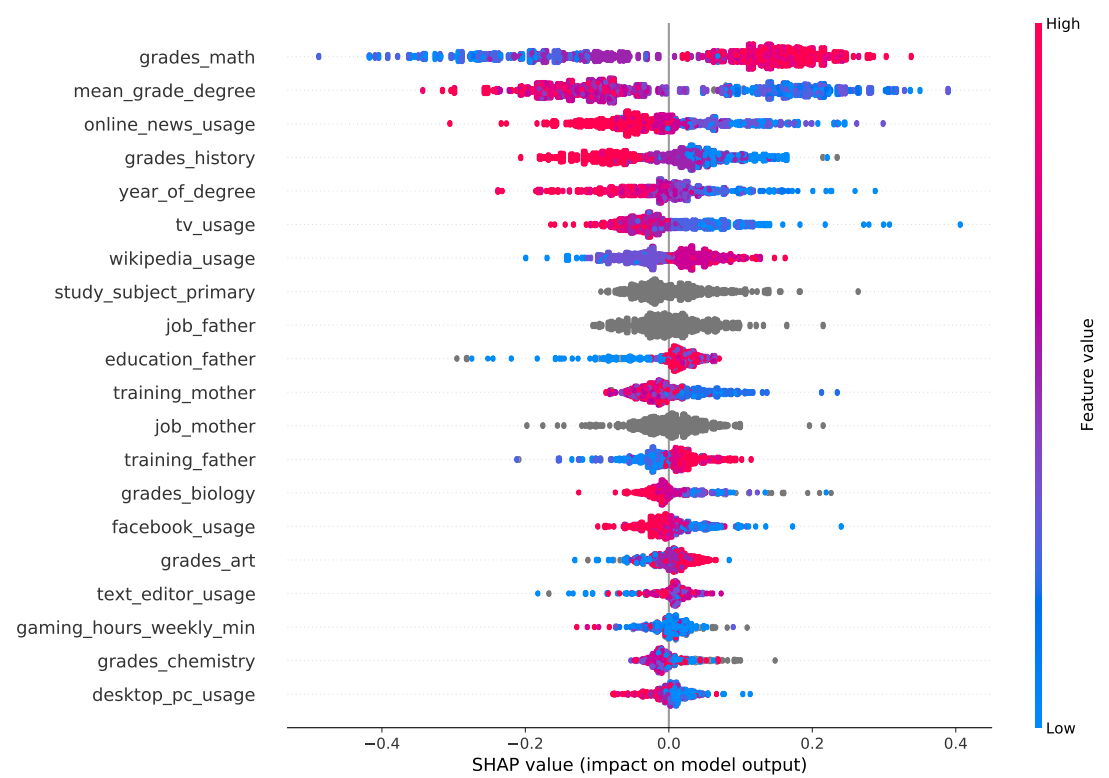

Fig 5. Summary plot of the approx. Shapeley values, that is, the density of the marginal contributions of the features in the GBDT model that uses only the socio-demographic features. Red denotes high feature values, whereas blue indicates low feature values, and grey show categorical values or missing values that cannot be assigned a feature value.

might be too low to thoroughly study microsaccade-related features. In addition, current literature reports inconsistent findings with regard to the underlying nature of microsaccades. While the majority of published papers consider this type of eye movements involuntary, others show that microsaccades can easily be triggered externally, e.g., [59,60, and thus raising the question of how to interpret microsaccades.

Pupil Diameter As shown in Table 1, we found a significant difference in the mean pupil diameter during problem solving as a function of items that were correctly solved vs. those answered incorrectly. Chen and Epps [11] report a smaller pupil response for tasks that overload participants as compared to tasks that participants successfully perform with very high load. The smaller pupil diameter that we observed for items that were answered incorrectly might reflect this cognitive overload.

\section{A machine learning perspective on the data}

We trained three GBDT models (i.e., using the eye-movement features, the socio-demographic information, and all 85 features) from the TüEyeQ data set to investigate the following questions:

Q1 Are the features related to eye-movements informative enough for predicting the success of a participant in solving a given CFT item?

Q2 If the previous question can be positively confirmed, is the information contained in the eye-movement features complementary to the information contained in the socio-demographic features? Or, more specifically, is a predictive model developed on both types of features, i.e., the eye-movement and the socio-demographic features, more discriminative than the models developed on the single subgroups of features? 


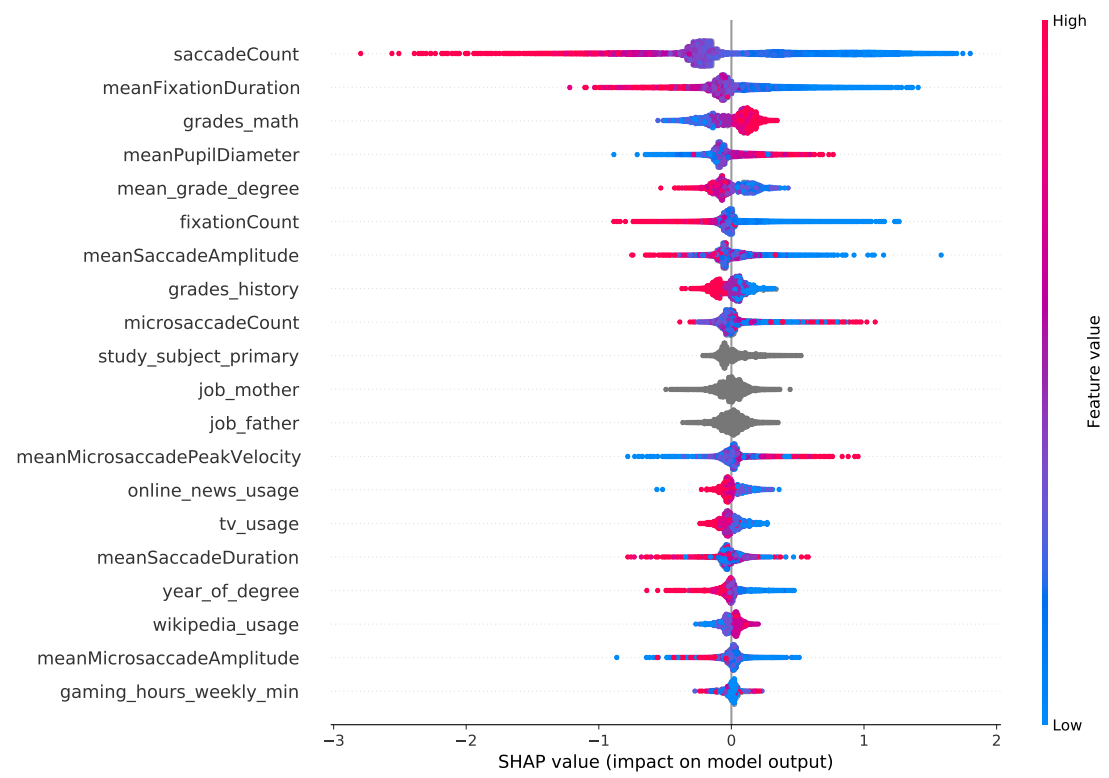

Fig 6. Summary plot of the approx. Shapeley values, that is, the density of the marginal contributions of the features in the GBDT model that uses only eye related and socio-demographic features. Red denotes high feature values, whereas blue indicates low feature values, and grey show categorical values or missing values that cannot be assigned a feature value.

The results from the GBDT model trained on only ten eye-movement features show that information contained in the eye movements is very discriminative (ROC-AUC of 0.63 as shown in Fig. 3ap. Our findings regarding Q1 are well in line with related literature, showing, thus, a significant association between eye-movement properties and fluid intelligence 28. Going beyond previous research which investigated a subset of these features in a rather fragmented way based on small sample sizes, our findings are based on a considerably larger sample size and sophisticated machine learning algorithms. With regard to Q2, we found socio-demographic information as captured by the GBDT model developed on the remaining 75 socio-demographic features to be less predictive (Fig. 3b ROC-AUC of 0.56) than the GBDT model on the eye-movement features. The machine learning model reveals even better predictive performance once all socio-demographic and eye-movement-related features are included, which confirms our assumption that information contained in these feature subsets is complementary and can be combined to significantly improve the predictive performance on whether a CFT item will be solved correctly by an individual.

Our explainability model confirmed that the eye-movement features with the most significant differences between both groups (task solved correctly vs. task not solved), i.e., saccadeCount, meanPupilDiameter, meanFixationDuration, and fixation count, have the highest impact for classification. Interestingly, multiple features derived from the microsaccades were revealed to significantly impact the model's prediction. However, we did not find any significant differences between the microsaccade-related features with regard to the items that were solved vs. those that were not solved, which might be also related to the fact that the sampling rate of our eye-tracking devices $(250$ $\mathrm{Hz}$ ) does not allow to capture fine-grained information on microsaccades. Figure 4 shows that the features microsaccadeCount and meanMicrosaccadeAmplitude have a high impact for classification to the positive class (i.e., item solved correctly). In 
contrast, meanMicrosaccadePeakVelocity has a high impact for classification to the negative class. Further research is needed to investigate the manifestation of fluid intelligence on microsaccade-related features and their causality.

The results of our explainability analysis regarding socio-demographic factors are in line with previous research in the literature $61-63$. Our analyses show a positive association between parental education level and occupation and an individual's performance on the CFT. Furthermore, students' academic background was also associated with performance on the CFT, confirming the findings in existing literature as well 65.

Importantly, our results show that a model that combines eye-movement and socio-demographic features performs significantly better than models trained exclusively on either of the two, suggesting that these two sources of information are complementary. This is supported by the explainability analysis that found 9 eye-related features and 11 socio-demographic features to be the 20 most predictive features for success in a given item of the CFT. Additionally, features that performed well in the combined model were also predictive in their respective single-category model, further backing the conclusion that eye movements and socio-demographic information contribute differential variance to the model.

\section{Limitations and future work}

Although the overall number of participants in our study is higher than that of related studies, it is important to note that all participants were university students, and as such, it is unclear whether the results will generalize to other populations. In our future work, we, therefore, aim to further investigate the contribution of individual differences on problem solving success in a more diverse population using eye-movement patterns 38, 39]. Here, we focused primarily on general eye-related features and investigated their predictive power on solving individual CFT items. As revealed by our explainability model, multiple features derived from the microsaccades significantly influenced the prediction of the machine learning model. Since the sampling rate of our eye-tracking devices was only $250 \mathrm{~Hz}$, these results can only be considered indications and require further investigations to gain insights on the relationship between microsaccades and problem solving success. Finally, there are additional sources of variance to consider that were not included here (but that could be added to the model), and thus, this work is a first step into using this approach to explain variance in problem solving success using a broad range of variables. Going one step further, counterfactual explanations [85, 86] could not only help identify important features/factors for predicting a person's performance on a problem, but also help develop individual strategies for efficient problem solving.

\section{Conclusion}

We found that specific eye-movement patterns are related to the ability of a participant to succeed in solving a given CFT item. Moreover, the eye-movement information is complementary to the socio-demographic information in predicting individual differences in problem solving success within the context of a standardized fluid intelligence test, suggesting that each source of information contributes important (but distinct) variance. Our method of analysis is based on a computational framework with machine learning and explainability at its core and thus, goes beyond purely correlational results. The sample size that we employed is considerably larger than what is typical in related research, which allowed the utilization of an extensive and rich feature set, while still maintaining the validity of our results, as demonstrated by our 
use of cross-validation. Overall, our computational framework that relies on a machine learning and explainability approach, might facilitate and thus contribute more in-depth investigations of a broad set of factors predicting individual differences in higher cognitive functions using large populations.

\section{Supporting information}

\section{Acknowledgments}

This research was supported as part of the LEAD Graduate School \& Research Network [GSC1028], which was funded within the framework of the Excellence Initiative of the German federal and state governments. Enkelejda Kasneci is a member of the Machine Learning Cluster of Excellence, EXC number 2064/1 - Project number 390727645.

\section{References}

1. Cattell Raymond B. Theory of fluid and crystallized intelligence: A critical experiment. Journal of educational psychology. 1963. 54:(1).

2. Weiß R. CFT 20-R.: Grundintelligenztest Skala 2. Manual. Göttingen: Hogrefe Verlag. 2006

3. Laurence PG, Mecca TP, Serpa A, Martin R, Macedo EC. Eye Movements and Cognitive Strategy in a Fluid Intelligence Test: Item Type Analysis. Frontiers in Psychology 9. 2018. 380

4. SoSci. SoSci Survey - the Solution for Professional Online Questionnaires. 2019, https://www.soscisurvey.de/

5. Engbert R, Kleigl, R. Microsaccades uncover the orientation of covert attention. Vision research. 2003 43(9):1035-1045.

6. Hafed ZM, Clark JJ. Microsaccades as an overt measure of covert attention shifts. Vision research. 2002 42(22):2533-2545.

7. Troncoso XG, Macknik SL, Martinez-Conde, S. Microsaccades counteract perceptual filling-in. Journal of vision. 2008 8(14):15-15.

8. Valsecchi M, Turatto M. Microsaccadic responses in a bimodal oddball task. Psychological research. 2009 73(1):23-33.

9. Siegenthaler EC, Francisco MM, Michael B, Di Stasi LL, Otero-Millan J, Sonderegger A, Groner R, Macknik S, Martinez-Conde, S. Task difficulty in mental arithmetic affects microsaccadic rates and magnitudes. European Journal of Neuroscience. 2014 39(2):287-294.

10. Appel T, Sevcenko N, Wortha F, Tsarava K, Moeller K, Ninaus M, Kasneci E, Gerjets P. Predicting Cognitive Load in an Emergency Simulation Based on Behavioral and Physiological Measures. 2019 International Conference on Multimodal Interaction. 2019.

11. Siyuan C, Julien E. Using Task-Induced Pupil Diameter and Blink Rate to Infer Cognitive Load. Human-Computer Interaction 2014 29:(4)390-413. 
12. Irwin DE, Thomas LE. Eyeblinks and cognition. Tutorials in visual cognition. 2010, 121-141, Psychology Press New York, NY

13. Rayner, K. Eye movements in reading and information processing: 20 years of research. Psychological bulletin. 1998 124(3):372.

14. Inamdar S, Pomplun M. Comparative search reveals the tradeoff between eye movements and working memory use in visual tasks. Proceedings of the annual meeting of the cognitive science society. 2003

15. He J, McCarley JS. Executive working memory load does not compromise perceptual processing during visual search: Evidence from additive factors analysis. Attention, Perception, \& Psychophysics. 2010 72(2):308-316.

16. Krejtz K, Duchowski AT, Niedzielska A, Biele C, Krejtz I. Eye tracking cognitive load using pupil diameter and microsaccades with fixed gaze. PloS one. 2018 13(9):e0203629.

17. Friedman JH. Stochastic gradient boosting. Computational statistics \& data analysis. 2002 38(4):367-378. Elsevier.

18. Jaeggi SM, Buschkuehl M, Jonides J, Perrig WJ. Improving fluid intelligence with training on working memory. Proceedings of the National Academy of Sciences. 2008 105(19):6829-6833.

19. Conway ARA, Cowan N, Bunting MF, Therriault DJ, Minkoff SRB. A latent variable analysis of working memory capacity, short-term memory capacity, processing speed, and general fluid intelligence. Intelligence. 2002 30(2):163-183

20. Kosinski M, Bachrach Y, Kasneci G, Van-Gael J, Graepel T. Crowd IQ: Measuring the intelligence of crowdsourcing platforms. Proceedings of the 4th annual ACM web science conference. 2012 151-160

21. Borisov V, Kasneci E, Kasneci G. Robust cognitive load detection from wrist-band sensors. Computers in Human Behavior Reports, 2021 4:100116. Elsevier

22. Gao H, Lu Z, Demberg V, Kasneci E. The Index of Cognitive Activity Predicts Cognitive Processing Load in Linguistic Task. In EMICS '21: ACM CHI '21 Workshop on Eye Movements as an Interface to Cognitive State, May 14, 2021. Yokohama, Japan. ACM, New York, NY, USA

23. Bachrach Y, Graepel T, Kasneci G, Kosinski M, Van Gael J. Crowd IQ: aggregating opinions to boost performance. Proceedings of the 11th International Conference on Autonomous Agents and Multiagent Systems. 2012 pp. 535-542

24. Lundberg SM, Lee SI. A unified approach to interpreting model predictions. Advances in neural information processing systems. 2017 pp. $4765-4774$

25. Lundberg SM, Erion GG, Lee SI. Consistent individualized feature attribution for tree ensembles. arXiv preprint arXiv:1802.03888. 2018

26. Ribeiro MT, Singh S, Guestrin C. "Why should I trust you?" Explaining the predictions of any classifier. Proceedings of the 22nd ACM SIGKDD international conference on knowledge discovery and data mining. 2016 pp.1135-1144

27. Salvucci DD, Goldberg JH. Identifying fixations and saccades in eye-tracking protocols. Proceedings of the 2000 Symposium on Eye tracking Research \& Applications. 2000 pp. $71-78$ 
28. Sargezeh BA, Ayatollahi AD, Mohammad R. Investigation of eye movement pattern parameters of individuals with different fluid intelligence. Experimental brain research. 2019 237(1):15-28. Springer

29. Leigh RJ, Zee DS. The neurology of eye movements. 2015 OUP USA

30. Lang F, Kammerer Y, Oschatz K, Stürmer K, Gerjets, P. The role of beliefs regarding the uncertainty of knowledge and mental effort as indicated by pupil dilation in evaluating scientific controversies. International Journal of Science Education. 2020 42(3):350-371. Taylor \& Francis

31. Scharinger C, Kammerer Y, Gerjets P. Pupil dilation and EEG alpha frequency band power reveal load on executive functions for link-selection processes during text reading. PloS one. 2015 10(6):e0130608.

32. Appel T, Gerjets P, Hoffman S, Moeller K, Ninaus M, Scharinger C, Sevcenko N, Wortha F, Kasneci E. Cross-task and Cross-participant Classification of Cognitive Load in an Emergency Simulation Game. IEEE Transactions on Affective Computing. 2021 IEEE

33. Castner N, Appel T, Eder T, Richter J, Scheiter K, Keutel C, Hüttig F, Duchowski A, Kasneci E. Pupil diameter differentiates expertise in dental radiography visual search. PloS one. 2020 15(5):e0223941

34. Friedman JH. Stochastic gradient boosting. Computational statistics \& data analysis. 2002. 38(4):367-378

35. Chen T, Guestrin C. XGBoost: A scalable tree boosting system. Proceedings of the 22nd acm sigkdd international conference on knowledge discovery and data mining. 2016 pp. $785-794$

36. Kuncheva LI, Whitaker CJ. Measures of diversity in classifier ensembles and their relationship with the ensemble accuracy. Machine learning. 2003 51(2):181-207. Springer

37. Rokach L. Ensemble-based classifiers. Artificial intelligence review. 2010 33(1):1-39: Springer

38. Kübler TC, Rothe C, Schiefer U, Rosenstiel W, Kasneci, E. SubsMatch 2.0: Scanpath comparison and classification based on subsequence frequencies. Behavior research methods. 2017 49(3):1048-1064

39. Castner N, Kasneci E, Kübler T, Scheiter K, Richter J, Eder T, Hüttig F, Keutel C. Scanpath comparison in medical image reading skills of dental students: distinguishing stages of expertise development. In Proceedings of the 2018 ACM Symposium on Eye Tracking Research Applications. 2018 pp. 1-9.

40. Hayes TR, Petrov AA, Sederberg PB. A novel method for analyzing sequential eye movements reveals strategic influence on Raven's Advanced Progressive Matrices. Journal of Vision. 2011 11(10):10-10. The Association for Research in Vision and Ophthalmology

41. Hayes TR, Petrov AA, Sederberg PB. Do we really become smarter when our fluid-intelligence test scores improve? Intelligence. 2015 48:1-14. Elsevier

42. Hayes TR, Petrov AA. Pupil diameter tracks the exploration-exploitation trade-off during analogical reasoning and explains individual differences in fluid intelligence. Journal of Cognitive Neuroscience. 2016 28(2):308-318 
43. Van Der Meer E, Beyer R, Horn J, Foth M, Bornemann B, Ries J, Kramer J, Warmuth E, Heekeren HR, Wartenburger I. Resource allocation and fluid intelligence: Insights from pupillometry. Psychophysiology. 2010 47(1):158-169. Wiley Online Library

44. Tsukahara JS, Harrison T, Engle RW. The relationship between baseline pupil size and intelligence. Cognitive psychology. 2016 91:109-123

45. Bornemann B, Foth M, Horn J, Ries J, Warmuth E, Wartenburger I, van der Meer E. Mathematical cognition: individual differences in resource allocation. ZDM. 2010 42(6):555-567. Springer

46. Gottfredson LS. Why g matters: The complexity of everyday life. Intelligence. 1997 24(1):79-132 Elsevier

47. Jaeggi SM, Buschkuehl M, Jonides J, Shah P. Short-and long-term benefits of cognitive training. Proceedings of the National Academy of Sciences. 2011 108(25):10081-10086. National Acad Sciences

48. Halford GS, Cowan N, Andrews G. Separating cognitive capacity from knowledge: A new hypothesis. Trends in cognitive sciences. 2007 11(6):236-242. Elsevier

49. Sheppard LD, Vernon PA. Intelligence and speed of information-processing: A review of 50 years of research. Personality and individual differences. 2008 44(3):535-551. Elsevier

50. Vakil E, Lifshitz-Zehavi H. Solving the Raven Progressive Matrices by adults with intellectual disability with/without Down syndrome: Different cognitive patterns as indicated by eye-movements. Research in Developmental Disabilities. $201233(2): 645-654$

51. Vigneau F, Caissie AF, Bors DA. Eye-movement analysis demonstrates strategic influences on intelligence. Intelligence. 2006 34(3):261-272. Elsevier

52. Shapley LS. A value for n-person games. Contributions to the Theory of Games. 1953 2(28):307-317

53. Haug J, Zürn S, El-Jiz P, Kasneci G. On baselines for local feature attributions. arXiv preprint arXiv:2101.00905. 2021.

54. Sigman MC, Sarale E, Beckwith L. Why does infant attention predict adolescent intelligence? Infant Behavior and Development. 1997 20(2):133-140. Elsevier

55. Meghanatha RN, van Leeuwen C, Nikolaev AR. Fixation duration surpasses pupil size as a measure of memory load in free viewing. Frontiers in Human Neuroscience. 2015 8:1063

56. Houtkamp R, Roelfsema PR. The effect of items in working memory on the deployment of attention and the eyes during visual search. Journal of Experimental Psychology: Human Perception and Performance. 2006 32(2):423. American Psychological Association

57. Fatima SS, Wooldridge M, Jennings NR. A linear approximation method for the Shapley value. Artificial Intelligence. 2008 172(14):1673-1699

58. Castro J, Gómez D, Tejada J. Polynomial calculation of the Shapley value based on sampling. Computers \& Operations Research. 2009 36(5):1726-1730. Elsevier 
59. Willeke KF, Tian X, Buonocore A, Bellet J, Ramirez-Cardenas A, Hafed ZM. Memory-guided microsaccades. Nature communications. 2019 10(1):1-14. Nature Publishing Group

60. Watanabe M, Matsuo Y, Zha L, Munoz DP, Kobayashi Y. Fixational saccades reflect volitional action preparation. Journal of neurophysiology. 2013 110(2):522-535. American Physiological Society Bethesda, MD

61. Murphy R, Cassimjee N, Schur C. Influence of socio-demographic factors on SRAVEN performance. Journal of Psychology in Africa. 21(1):91-101. Taylor \& Francis

62. Vista AD, Grantham TC. Effects of parental education level on fluid intelligence of Philippine public school students. Journal of Psychoeducational Assessment. 2010 28(3):236-248. Sage Publications Sage CA: Los Angeles, CA

63. Deckers T, Falk A, Kosse F, Pinger P, Schildberg-Hörisch, H. Socio-economic status and inequalities in children's IQ and economic preferences. DICE Discussion Paper. $2017=$ http://hdl.handle.net/10419/171935 Düsseldorf Institute for Competition Economics (DICE)

64. van der Wel P, van Steenbergen H. Pupil dilation as an index of effort in cognitive control tasks: A review. Psychonomic bulletin \& review. 2018, 25(6):2005-2015. Springer

65. Kaufman AS, Kaufman JC, Liu X, Johnson CK. How do Educational Attainment and Gender Relate to Fluid Intelligence, Crystallized Intelligence, and Academic Skills at Ages 22-90 Years?. Archives of Clinical Neuropsychology. 2009 24(2):153-163

66. Ahern S, Beatty J. Pupillary responses during information processing vary with Scholastic Aptitude Test scores., Science. 1979 205(4412):1289-1292. American Association for the Advancement of Science

67. Rindermann H, Flores-Mendoza C, Mansur-Alves M. Reciprocal effects between fluid and crystallized intelligence and their dependence on parents' socioeconomic status and education. Learning and Individual Differences. 2010 20(5):544-548. Elsevier

68. Kasneci E, Kasneci G, Appel T, Haug J, Wortha F, Tibus M, Trautwein U, Gerjets, P. TüEyeQ: A rich IQ test performance data set with eye movement, educational and socio-demographic information. Harvard Dataverse. 2020 https://doi.org/10.7910/DVN/JGOCKI

69. Logie R. Human Cognition: Common Principles and Individual Variation. Journal of Applied Research in Memory and Cognition. 2018 7(4):471-486

70. Hackman DA, Farah M, Meaney MJ. Socioeconomic status and the brain: mechanistic insights from human and animal research. Nature reviews neuroscience. 2010 11(9):651-659. Nature Publishing Group

71. Brito NH, Noble KG. Socioeconomic status and structural brain development. Frontiers in neuroscience. 2014 8:276. Frontiers

72. Zhang Q, Wang C, Zhao Q, Yang L, Buschkuehl M, Jaeggi SM. The malleability of executive function in early childhood: Effects of schooling and targeted training. Developmental science. 2019 22(2):e12748. Wiley Online Library 
73. Hillman CH, Erickson KI, Kramer AF. Be smart, exercise your heart: exercise effects on brain and cognition. Nature reviews neuroscience. 2008 9(1):58-65.

Nature Publishing Group

74. Bavelier D, Green CS, Pouget A, Schrater P. Brain plasticity through the life span: learning to learn and action video games. Annual review of neuroscience. 2012 35:391-416. Annual Reviews

75. Waris O, Jaeggi SM, Seitz AR, Lehtonen M, Soveri A, Lukasik KM, Öderström U, Hoffing RAC, Laine M. Video gaming and working memory: A large-scale cross-sectional correlative study. Computers in human behavior. 2019 97:94-103. Elsevier

76. Slevc LR, Davey NS, Buschkuehl M, Jaeggi SM. Tuning the mind: Exploring the connections between musical ability and executive functions. Cognition. 2016 152:199-211. Elsevier

77. Appel T, Scharinger C, Gerjets P, Kasneci E. Cross-subject workload classification using pupil-related measures. Proceedings of the 2018 ACM Symposium on Eye Tracking Research \& Applications. 2018 1-8

78. Curie A, Brun A, Cheylus A, Reboul A, Nazir T, Bussy G, Delange K, Paulignan Y, Mercier S, David A. A novel analog reasoning paradigm: new insights in intellectually disabled patients. PloS one. 2016 11(2):e0149717

79. Kasneci E, Kasneci G, Appel T, Haug J, Wortha F, Tibus M, Trautwein U, Gerjets P. TüEyeQ, a rich IQ test performance data set with eye movement, educational and socio-demographic information. Scientific Data. 2021 8(1):1-14. Nature Publishing Group

80. Hill BD, Aita SL. The positive side of effort: A review of the impact of motivation and engagement on neuropsychological performance. Applied Neuropsychology: Adult. 2018 25(4):312-317. Taylor \& Francis

81. Duckworth AL, Quinn PD, Lynam DR, Loeber R, Stouthamer-Loeber M. Role of test motivation in intelligence testing. Proceedings of the National Academy of Sciences. 2011 108(19):7716-7720. National Acad Sciences

82. Donaldson SI, Grant-Vallone EJ. Understanding self-report bias in organizational behavior research. Journal of Business and Psychology. 2002 17(2):245-260. Springer

83. Snow RE. Aptitude processes. Aptitude, learning, and instruction. 1980 1:27-63

84. Bethell-Fox CE, Lohman DF, Snow RE. Adaptive reasoning: Componential and eye movement analysis of geometric analogy performance. Intelligence. 1984 8(3):205-238. Elsevier

85. Pawelczyk M, Broelemann K, Kasneci, G. Learning model-agnostic counterfactual explanations for tabular data. Proceedings of The Web Conference. 2020 pp.3126-3132

86. Pawelczyk M, Bielawski S, van den Heuvel J, Richter T, Kasneci G. Carla: a python library to benchmark algorithmic recourse and counterfactual explanation algorithms. arXiv preprint arXiv:2108.00783. 2021 


\begin{tabular}{|c|c|c|c|}
\hline Variable Nr. & Feature & Description & Encoding \\
\hline 1 & TaskID & Unique identifier for every task & String, CFT-block-related task id \\
\hline 2 & subject & Unique identifier for every participant & String-based id \\
\hline 3 & age & The age of a participant & categorical \\
\hline 4 & gender & The gender of a participant, i.e. male, female, unknown & categorical \\
\hline 5 & handedness & Indicates whether the participant is right-handed or left-handed & binary \\
\hline 6 & native_german & This variable describes whether a participant is a native German & binary \\
\hline 7 & native_german_mother & Indicates whether the mother of the participant is a native German & binary \\
\hline 8 & native_language_mother & The native language of the participant's mother & categorical \\
\hline 9 & native_german_father & Indicates whether the father of the participant is a native German & binary \\
\hline 10 & native_language_father & The native language participant's father & categorical \\
\hline 11 & education_mother & The scholarly or professional education of the participant's mother & categorical \\
\hline 12 & education_father & The scholarly or professional education of the participant's father & categorical \\
\hline 13 & training_mother & The scholarly or professional training of the participant's mother & categorical \\
\hline 14 & training_father & The scholarly or professional training of the participant's father & categorical \\
\hline 15 & books & Indicates how many books are in the participant household & categorical \\
\hline 16 & job_mother & The profession of the participant's mother & categorical \\
\hline 17 & job_father & The profession of the participant's father & categorical \\
\hline 18 & year_of_degree & The year in which the final study degree was achieved by the participant & categorical \\
\hline 19 & mean_grade_degree & The average grade of the participant's final degree & continuous \\
\hline 20 & programming_experience & Indicates whether the participant has experience programming languages & binary \\
\hline 21 & smartphone_usage & Indicates the frequency of smartphone usage (range: never to daily) & categorical \\
\hline 22 & tablet_usage & Indicates the frequency of tablet usage (range: never to daily) & categorical \\
\hline 23 & notebook_usage & Indicates the frequency of notebook usage (range: never to daily) & categorical \\
\hline 24 & desktop_pc_usage & Indicates the frequency of desktop pc usage (range: never to daily) & categorical \\
\hline 25 & tv_usage & Indicates the frequency of tv usage (range: never to daily) & categorical \\
\hline 26 & text_editor_usage & Indicates the frequency of text editors usage (range: never to daily) & categorical \\
\hline 27 & spreadsheet_usage & Indicates the frequency of spreadsheet software usage (range: never to daily) & categorical \\
\hline 28 & presentation_software_usage & Indicates the frequency of presentation software usage (range: never to daily) & categorical \\
\hline 29 & email_usage & Indicates the frequency of email usage (range: never to daily) & categorical \\
\hline 30 & browser_usage & Indicates the frequency of web browser usage (range: never to daily) & categorical \\
\hline 31 & google_usage & Indicates the frequency of Google usage (range: never to daily) & categorical \\
\hline 32 & wikipedia_usage & Indicates the frequency of Wikipedia usage (range: never to daily) & categorical \\
\hline 33 & facebook_usage & Indicates the frequency of Facebook usage (range: never to daily) & categorical \\
\hline 34 & twitter_usage & Indicates the frequency of Twitter usage (range: never to daily) & categorical \\
\hline 35 & skype_usage & Indicates the frequency of Skype usage (range: never to daily) & categorical \\
\hline 36 & youtube_usage & Indicates the frequency of Youtube usage (range: never to daily) & categorical \\
\hline 37 & ebay_usage & Indicates the frequency of Eabay usage (range: never to daily) & categorical \\
\hline 38 & amazon_usage & Indicates the frequency of Amazon usage (range: never to daily) & categorical \\
\hline 39 & online_news_usage & Indicates the frequency of online news usage (range: never to daily) & categorical \\
\hline 40 & online_banking_usage & Indicates the frequency of online banking usage (range: never to daily) & categorical \\
\hline 41 & gaming_adventure & Indicates whether the participant primarily plays adventure games & binary \\
\hline 42 & gaming_action & Indicates whether the participant primarily plays action games & binary \\
\hline 43 & gaming_first_person_shooter & Indicates whether the participant primarily plays first person shooter games & binary \\
\hline 44 & gaming_casual & Indicates whether the participant primarily plays casual games & binary \\
\hline 45 & gaming_mmo & $\begin{array}{l}\text { Indicates whether the participant primarily plays Massive Multiplayer Online } \\
\text { games }\end{array}$ & binary \\
\hline 46 & gaming_racing & Indicates whether the participant primarily plays racing games & binary \\
\hline 47 & gaming_rpg & Indicates whether the participant primarily plays Role Playing Games games & binary \\
\hline 48 & gaming_simulation & Indicates whether the participant primarily plays simulation games & binary \\
\hline 49 & gaming_sports & Indicates whether the participant primarily plays sports games & binary \\
\hline 50 & gaming_strategy & Indicates whether the participant primarily plays strategy games & binary \\
\hline 51 & smoking & Indicates whether the participant is a smoker & binary \\
\hline 52 & excessive_drinking & Indicates whether the participant is an excessive drinker & binary \\
\hline 53 & grades_math & The participant's final math grade (German Abitur) & continuous \\
\hline 54 & grades_german & The participant's final German grade (German Abitur) & continuous \\
\hline 55 & grades_biology & The participant's final biology grade (German Abitur) & continuous \\
\hline 56 & grades_physics & The participant's final physics grade (German Abitur) & continuous \\
\hline 57 & grades_chemistry & The participant's final chemistry grade (German Abitur) & continuous \\
\hline 58 & grades_geography & The participant's final geography grade (German Abitur) & continuous \\
\hline 59 & grades_history & The participant's final history grade (German Abitur) & continuous \\
\hline 60 & grades_art & The participant's final art grade (German Abitur) & continuous \\
\hline 61 & gaming_hours_weekly_min & The minimum hours the participant spends gaming per week & continuous \\
\hline 62 & gaming_hours_weekly_max & The maximum hours the participant spends gaming per week & continuous \\
\hline 63 & leisure_simple_entertainment & $\begin{array}{l}\text { Indicates whether the participant's leisure activity involves simple entertain- } \\
\text { ment }\end{array}$ & binary \\
\hline 64 & leisure_mental_activity & Indicates whether the participant's leisure activity involves mental activity & binary \\
\hline 65 & leisure_sports_exercise & $\begin{array}{l}\text { Indicates whether the participant's leisure activity involves sports and exer- } \\
\text { cise }\end{array}$ & binary \\
\hline 66 & leisure_music & Indicates whether the participant's leisure activity involves music & binary \\
\hline 67 & leisure_art & Indicates whether the participant's leisure activity involves art & binary \\
\hline 68 & leisure_dance & Indicates whether the participant's leisure activity involves dance & binary \\
\hline 69 & leisure_hobbies & $\begin{array}{l}\text { Indicates whether the participant's leisure activity involves hobbies (e.g. } \\
\text { DIY) }\end{array}$ & binary \\
\hline 70 & leisure_play_games & $\begin{array}{l}\text { Indicates whether the participant's leisure activity involves playing (video-) } \\
\text { games }\end{array}$ & binary \\
\hline 71 & leisure_relaxation & Indicates whether the participant's leisure activity involves relaxation & binary \\
\hline 72 & leisure_social_activity & Indicates whether the participant's leisure activity involves social activities & binary \\
\hline 73 & leisure_humanitarian_services & $\begin{array}{l}\text { Indicates whether the participant's leisure activity involves humanitarian } \\
\text { work }\end{array}$ & binary \\
\hline 74 & leisure_nature_activities & $\begin{array}{l}\text { Indicates whether the participant's leisure activity involves nature/outdoor } \\
\text { activities }\end{array}$ & binary \\
\hline 75 & leisure_travel_tourism & $\begin{array}{l}\text { Indicates whether the participant's leisure activity involves travel and } \\
\text { tourism }\end{array}$ & binary \\
\hline 76 & study_subject_primary & The primary study subject category of the participant & categorical \\
\hline 77 & study_subject_secondary & The secondary study subject category of the participant & categorical \\
\hline 78 & cft_sum_full & The aggregated CFT score of the participant & continuous \\
\hline 79 & cft_task & Indicates whether the participant solved the task correctly & binary \\
\hline 80 & fixationCount & The number of fixations performed by a participant during a test item & continuous \\
\hline 81 & meanFixationDuration & The mean duration of fixations performed by a participant during a test item & continuous \\
\hline 82 & saccadeCount & The number of saccades performed by a participant during a test item & continuous \\
\hline 83 & meanSaccadeAmplitude & $\begin{array}{l}\text { The mean amplitude of saccades performed by a participant during a test } \\
\text { item }\end{array}$ & continuous \\
\hline 84 & meanSaccadeDuration & The mean duration of saccades performed by a participant during a test item & continuous \\
\hline 85 & microsaccadeCount & The number of microsaccades that occured during a test item & continuous \\
\hline 86 & meanMicrosaccadeAmplitude & The mean amplitude of microsaccades during a test item & continuous \\
\hline 87 & meanMicrosaccadeDuration & The mean duration of microsaccades during a test item & continuous \\
\hline 88 & meanMicrosaccadePeakVelocity & The mean peak velocity of microsaccades during a test item & continuous \\
\hline 89 & meanPupilDiameter & The mean pupil diameter of a participant during a test item & continuous \\
\hline
\end{tabular}

Table 2. Description and encoding of all performance-related, educational and socio-demographic features in the order of their appearance in the csv file as provided by the TüEyeQ data set (available through the Harvard Dataverse Repository under https://doi.org/10.7910/DVN/JGOCKI). 